\title{
Integral field observations of distant cluster galaxies
}

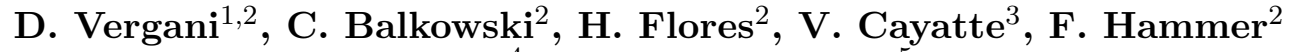 \\ S. $\mathrm{Mei}^{4}$, and J.P. Blakeslee ${ }^{5}$ \\ ${ }^{1}$ INAF - IASF Milano, via Bassini 15, 20133 Milano, Italy \\ email: daniela@lambrate.inaf.it
}

${ }^{2}$ Obs. de Paris, Sec. de Meudon, GEPI, 5 place Jules Janssen, F-92195 Meudon Cedex, France

${ }^{3}$ Obs. de Paris, Sec. de Meudon, LUTH, 5 place Jules Janssen, F-92195 Meudon Cedex, France

${ }^{4}$ Dep. of Physics and Astronomy, Johns Hopkins University, Baltimore, MD 21218

${ }^{5}$ Dep. of Physics and Astronomy, Washington State University, Pullman, WA 99164-2814

\begin{abstract}
We have used the FLAMES multi-integral field unit system of the European Southern Observatory (VLT) centered on the cluster MS0451.6-0305 at $z=0.5386$ to obtain the spatially resolved kinematics of the cluster members. The spectral data are supported by HST/ACS images that provide immediate morphological information of the cluster galaxies. The relevant structural parameters such as inclination, size, and orientation derived from optical high angular resolution images are compared with those derived from the kinematics. Our final goals are: 1 . to derive the Tully-Fisher relation for cluster galaxies with regular kinematics. 2. to obtain the dynamical masses from resolved kinematics and stellar masses from optical images to be compared with local measurements.
\end{abstract}

Keywords. instrumentation: spectrographs, galaxies: clusters: individual (MS0451.6-0305)

\section{Context}

Being the largest gravitationally bound objects in the Universe, cluster galaxies are excellent tools to explore the distant Universe and to constrain cosmological models.

Integral field spectroscopic studies appear to be a pre-requisite to sample the whole velocity field of individual galaxies, and to limit uncertainties related to observations (e.g. the major-axis determination, slit misalignment) and internal processes (e.g. kinematic disturbances due to interactions). In this respect, the ability of VLT/FLAMES-IFU to provide simultaneously 15 resolved velocity fields opens the possibility to study in detail kinematics in distant clusters.

We observe the massive X-ray cluster MS0451.6-0305 at $z=0.5386$ tracing the resolved kinematics of the [OII] $3727 \AA$ emission line with VLT/FLAMES-IFU. Galaxies in the cluster have shown very different kinematic and morphological behaviors. They present anomalies at some extent, i.e. warps, out-of-plane star formation, stripping/merging events. We derived the rotation curves for eight members which represent the best dataset to perform studies on the dynamic evolution of galaxies, especially in rich environments where mechanisms of ram-pressure stripping and mergers occur more frequently.

\section{Acknowledgements}

DV acknowledges supports through a Euro3D RTN on Integral Field Spectroscopy (No. HPRN-CT-2002-00305) and the Marie Curie ERG grant (No. MERG-CT-2005-021704), funded by the European Commission. DV also acknowledges the support of the IAU Grant to attend the IAU conference. 